\title{
La modernización de los contenidos y métodos de enseñanza: re- flexiones sobre la Escuela Nueva en Colombia ${ }^{1}$
}

\author{
Rafael Ríos Beltrán ${ }^{2}$ \\ Instituto de Educación y Pedagogía de la Universidad del Valle \\ rafael.rios@correounivalle.edu.co \\ Martha Yanet Cerquera Cuellar ${ }^{3}$ \\ Instituto de Educación y Pedagogía de la Universidad del Valle \\ maryane_c@hotmail.com
}

Recepción: 06/05/2013

Evaluación: 03/11/2013

Aceptación: 13/12/2013

Artículo de Reflexión

DOI: http: / / dx.doi.org/ 10.9757/ Rhela.22 / 07

Problema común a todas las naciones jóvenes es el de salvar, en reducido espacio de
tiempo, el camino que los estados de la vieja civilización han recorrido a través de
un proceso. Colombia es uno de aquellos países. Apenas obtenida su independencia,
encontrase frente a la necesidad de elevar el nivel de su cultura desde la situación
primitiva en que se hallaba hasta las alturas que demandan los tiempos modernos. ${ }^{4}$

\section{RESUMEN}

La Escuela Nueva en Colombia significó la apertura hacia un proceso de modernización de los contenidos y de reforma de los métodos de enseñanza. Desde una perspectiva histórica, el artículo describe este proceso por medio de los métodos que mayores transformaciones operaron en los sistemas y métodos de enseñanza en el saber pedagógico colombiano durante la primera mitad del siglo XX, a saber: los Centros de Interés, del médico belga Ovidio Decroly y el Método de Proyectos derivado de la pedagogía activa del norteamericano John Dewey. Los maestros en formación y egresados de las escuelas normales hicieron uso de estos dos sistemas de enseñanza para globalizar la enseñanza y para romper con el antiguo aislamiento de las diferentes asignaturas. Sus tesis o monografías de grado constituyen un campo documental invaluable para comprender la complejidad de las prácticas de apropiación de este acontecimiento pedagógico en Colombia.

\section{Palabras clave: Revista Historia de la Educación Latinoamericana, método de enseñanza, Escuela Nueva en Colombia, modernidad educativa, historia de la enseñanza, saber pedagógico.}

1 Este artículo es el resultado de los siguientes proyectos de investigación: 1. La apropiación de la escuela nueva en el saber pedagógico colombiano: una mirada a las escuelas normales durante la primera mitad del siglo XX, financiado por Colciencias y el CODI de la Universidad de Antioquia. 2. La apropiación de la escuela nueva en el saber pedagógico colombiano: una mirada a la Escuela Normal de Institutores de Antioquia, 1925-1945, financiado por el CODI de la Universidad de Antioquia y la Escuela Normal Superior de Medellín.

2 Doctorado en Educación Universidad de Antioquia, actualmente está vinculado como profesor en el Instituto de Educación y Pedagogía de la Universidad del Valle, pertenece al Grupo "Historia de la práctica pedagógica”.

3 Magíster en Educación, actualmente estudiante del Doctorado Interinstitucional de Educación de la Universidad del Valle, pertenece al Grupo de Investigación en Pedagogía y Práctica pedagógica.

4 Gonzalo, Bravo. "Consideraciones generales acerca del maestro" Tesis de grado para optar al título de maestro. Escuela Normal de Institutores de Antioquia. Medellín, 1939. 
The modernization of the contents and methods of education: Reflections on the new school in Colombia

ABSTRACT

The New School in Colombia meant the opening towards a process of modernization of the contents and of reform of the teaching methods. From a historical perspective, the article describes this process across the methods that produced the main changes in the systems and teaching methods in the Colombian pedagogical knowledge during the first half of the XXth century. To know: the Centers of Interest, of the Belgian doctor Ovidio Decroly and the Project method derived from the active pedagogics of the North American John Dewey. The teachers in development and ex-students made use of these two systems of education to encompass the education and to break with the ancient isolation of the different subjects. Its dissertation or monographs of grade constitute a field documentary invaluable to understand the complexity of the practices of appropriation of this pedagogic event in Colombia.

Key words: Journal History of the Latin-American Education, method of education, New School in Colombia, educational modernity, history of the education, to know pedagogically.

\section{A modernização dos conteúdos e métodos de ensino: Reflexões sobre a escola nova na Colômbia}

T1 RESUMO

A Escola Nova na Colômbia significou a abertura para um processo de modernização dos conteúdos e de reforma dos métodos de ensino. A partir de uma perspectiva histórica, o artigo descreve este processo através dos métodos que maiores transformações operaram nos sistemas e métodos de ensino no saber pedagógico colombiano durante a primeira metade do século $\mathrm{XX}$, a saber: os Centros de interesse, do médico belga Ovidio Decroly e o Método de Projetos derivado da pedagogia ativa do norte-americano John Dewey. Os professores em formação e egressos das escolas normais fizeram uso destes dois sistemas de ensino para globalizar o ensino e para romper com o antigo isolamento das diferentes disciplinas. Suas teses ou monografias de conclusão de curso constituem um campo documental inestimável para compreender a complexidade das práticas de apropriação deste acontecimento pedagógico na Colômbia.

Palavras-chave: Revista História da Educação Latino-americana, método de ensino, Escola Nova na Colômbia, modernidade educativa, história do ensino, saber pedagógico.

\section{INTRODUCCIÓN}

\section{En el saber pedagógico colombiano se pueden identificar cuatro tendencias mediante las cuales ocurrió el proceso de apropiación de las concepciones de la Escuela Nueva, ${ }^{5}$ a saber: a) escuela examinadora, la cual transformó}

5 Existen dos investigaciones muy importantes que, desde diferentes enfoques, analizaron el proceso de apropiación de la Escuela Nueva, tanto en el discurso como en la práctica pedagógica en nuestro país. La primera de ellas es la realizada por Javier Sáenz Obregón, Oscar Saldarriaga y Armando Ospina, Mirar la infancia: pedagogía, moral y modernidad en Colombia, (Bogotá, Medellín: Colciencias-Foro Nacional por Colombia-Ediciones Uniandes-Editorial Universidad de Antioquía, 1997). La segunda investigación es la tesis doctoral de la profesora Martha Cecilia Cortés, Modernización y Escuela Nueva en Colombia: 1914-1951, (Bogotá: Plaza \& Janes editores, 1999). Nuestra investigación no pretende desprenderse de los análisis ya realizados en los trabajos anteriormente citados sobre el proceso de apropiación de la Escuela Nueva en nuestro país, ya que estos hacen importantes análisis sobre las diferentes apropiaciones de la Escuela Nueva en Colombia, que sustentaron las reformas en la formación del magisterio, de los funcionarios de la educación pública y las reorientaciones de la escuela primaria, durante la primera mitad del siglo XX, tampoco quiere mirar con los mismos lentes este proceso, sino que sitúa en otro lugar su pregunta, pues analiza la apropiación de la Escuela Nueva en las instituciones formadoras de maestros, como un acontecimiento pedagógico complejo y variado que asume diversas características en relación con la didáctica y la enseñanza de las ciencias y los saberes. 
las escuelas en laboratorio de experimentación y medición. La Casa de Corrección de Menores de Fontidueño y la Escuela Especial Rafael Uribe Uribe en Antioquia representaron las primeras experiencias en donde se implementó el conjunto de prácticas de la pedagogía experimental en Colombia. En estas escuelas el régimen institucional estaba dirigido a examinar al niño, moral y orgánicamente, para profundizar en el diagnóstico sobre las causas de la anormalidad en el alumno. ${ }^{6}$ b) escuela defensiva, que asumió las escuelas como espacio para construir una raza culta y civilizada. Experiencia piloto en Colombia fue la del Departamento de Boyacá, liderada por Rafael Bernal, entre 1925 y 1930, la cual se centró en la transformación de la escuela tradicional en una escuela de defensa de la raza. El concepto reformador de la experiencia de Boyacá fue el de raza degenerada física, moral e intelectualmente, y estuvo focalizada a una población rural y mestiza e inspirada en las concepciones de Decroly respecto a la función de la educación como defensa de la infancia contra los peligros de la herencia y el ambiente. c) reforma de los métodos de enseñanza, y d) educación para la democracia, la cual hizo énfasis en los fines sociopolíticos de la educación pública, a la función social y política de la escuela, a su participación en la resolución de los problemas inmediatos de la sociedad y al desarrollo de la responsabilidad social de los alumnos. La primera experiencia en este sentido la tuvimos con el Gimnasio Moderno en Bogotá. ${ }^{7}$

En este artículo nos centraremos en la tercera tendencia relacionada con la reforma de los métodos de enseñanza por medio de las prácticas de escritura que ejercieron los maestros en formación con sus tesis o monografías para optar por título de maestro. Las tesis para optar por el título de institutor en las Escuelas Normales son documentos que reúnen elementos, huellas, enunciados que dan pistas sobre las reglas de formación de un discurso sobre la enseñanza de los diversos saberes en la escuela. Estos documentos, entre otros, elaborados por los maestros, hacen visible el campo enunciativo ${ }^{8}$ desde el cual se apropia la Escuela Nueva en Colombia, durante la primera mitad

6 Jaime, Botero. "La educación de los niños mentalmente anormales" (Tesis de grado, para optar al título de Institutor de maestro. Escuela Normal de Institutores de Antioquia. Medellín, 1940)

7 Humberto Quiceno, Javier Sáenz Obregón y Luis Arturo Vahos, “La instrucción y la educación pública en Colombia: 1903-1997”, en Modernización de los sistemas educativos iberoamericanos siglo XX, Tomo II, editado por Olga Lucia Zuluaga Garcés y Gabriela Ossenbach Sauter. (Bogotá: Editorial Magisterio, Colección Pedagogía e Historia, 2004), 138

8 Campo enunciativo designa el conjunto de todos aquellos enunciados formulados y postulados en otros discursos y que son admitidos como verdades demostradas, como también, a aquellos que son rechazados o han sido refutados. Este campo es el que permite introducir citas, referencias, autoridades, y que son utilizados para confirmar o validar observaciones. Sergio Albano, Michel Foucault. Glosario epistemológico, (Buenos Aires: Quadrata, 2007), 58. 
del siglo XX. Asumimos el análisis de la Escuela Nueva, y su apropiación en el saber pedagógico colombiano, como un acontecimiento pedagógico complejo y variado que asume diversas características en relación con la didáctica y la enseñanza de las ciencias y los saberes en la escuela primaria y secundaria. También observamos que en este proceso se produce una normalización del trabajo escolar por medio de los métodos de enseñanza. A un saber transmitido con meras palabras, debía aparecer un método que mostrara las cosas, o derivado de las cosas, por trato directo o por su manejo.

Varias son las herramientas conceptuales que orientaron el análisis. En primer lugar, la definición de pedagogía, entendida como "la disciplina que conceptualiza, aplica y experimenta los conocimientos referentes a la enseñanza de los saberes específicos, en diferentes culturas".$^{9}$ Esta definición subraya la especificidad de la pedagogía como un saber que tiene su propia historia, conceptos, prescripciones y objetos, el cual tiene un discurso propio, un sujeto específico de saber, y una institución específica de aplicación y experimentación. En segundo lugar, y a partir de esta definición, el maestro es concebido no simplemente como el sujeto de las prácticas de enseñanza, sino como el sujeto del saber pedagógico: como el sujeto en el que se completa la "teoría" y la "práctica". Aquí, el maestro como sujeto de saber toma posición frente a los enunciados que usa en su discurso. En tercer lugar, entendemos la Didáctica como el conjunto de conocimientos referentes al enseñar y aprender que conforman un saber. Este saber es una construcción posible a partir de las relaciones que se establecen entre pedagogía, ciencias, disciplinas y saberes. En consecuencia, en la Didáctica se localizan conceptos teóricos y conceptos operativos, tanto de la pedagogía como del campo científico o disciplinar, que impiden una asimilación de la Didáctica a meras fórmulas y recetas para enseñar un saber o como un conjunto de procedimientos generales para tener en cuenta en la enseñanza de cualquier ciencia o contenido científico. Los parámetros de las conceptualizaciones en la Didáctica se refieren a la forma de conocer o de aprender del hombre, a los conocimientos objeto de la enseñanza, a los procedimientos para enseñar, a la educación y a sus particularidades, condiciones o estrategias bajo las cuales debe ser enseñado y aprendido un saber específico. Finalmente, el concepto de apropiación fue una herramienta de análisis importante para entender que los saberes adoptados se incrustaron en nuestras propias

9 Olga Lucia Zuluaga, Pedagogía e historia, (Bogotá: Antrophos, 1999), 11. 
condiciones políticas y culturales. Esto explica que la apropiación no consiste solo en la recepción de saberes y métodos, sino también en la acción sobre lo que llega. ${ }^{10}$

El artículo se plantea desarrollar el análisis desde el siguiente interrogante: ¿Desde qué concepciones se configura la propuesta didáctica de la Escuela Nueva para transformar y modernizar los métodos de enseñanza de las ciencias y los saberes en la escuela? Bien sabemos que cualquier reforma educativa pasaba necesariamente por los institutores, y era preciso formarlos en las concepciones nuevas sobre materia de educación y enseñanza moderna. De acuerdo con esto, el presente escrito describe los dos métodos que apropiaron los maestros en formación y egresados de las escuelas normales, para la enseñanza de las ciencias y los saberes en la escuela: los Centros de Interés del belga Decroly y el Método de Proyectos del norteamericano John Dewey. Se cierra el artículo con el desarrollo de algunas reflexiones que apuntan a describir los efectos de la apropiación de la Escuela Nueva en nuestro país.

\section{La reforma de los métodos de enseñanza}

Con la emergencia de la Escuela Nueva durante la primera mitad del siglo $\mathrm{XX}$ en Colombia, el sistema pedagógico moderno de Pestalozzi ${ }^{11}$ comienza a ser visto como el proyecto a abandonar por su condición de tradicional y antiguo frente a la emergencia del proyecto de la Escuela Nueva, considerado ahora bajo el ropaje de lo nuevo respecto a la reforma de los métodos de enseñanza.

El desplazamiento de Lancaster a Pestalozzi, acontecido en el siglo XIX y de Pestalozzi a Decroly durante la tercera década del siglo XX, nos muestra una de las rutas por medio de la cual nos adentramos en el juego por la modernización de los contenidos de enseñanza en nuestro país.

La apropiación de la Escuela Nueva en Colombia no fue ajena a la clásica dualidad entre lo antiguo y lo moderno, liderada por los partidos políticos

10 Olga Lucia Zuluaga Garcés. "Prólogo” en Mirar la infancia: pedagogía, moral y modernidad en Colombia, 1903-1946, Volumen I, realizado por Javier Sáenz Obregón, Oscar Saldarriaga y Armando Ospina, X-x. (Medellín: Colciencias- Foro Nacional por Colombia-Uniandes-Universidad de Antioquía, 1997), XXI - XXV.

11 La pedagogía pestalozziana denominada también "objetiva" o "intuitiva" se recepciona por primera vez en Colombia entre los años de 1845 y 1847, como un procedimiento auxiliar para mejorar la enseñanza de la gramática y la matemática. 
(liberal-conservador). Además, los maestros en ejercicio y en formación en las escuelas normales fueron una pieza central en esta mirada longitudinal del proyecto de reforma. Sus reflexiones y ensayos sobre cómo enseñarían un saber o disciplina bajo los fundamentos de la Escuela Nueva, los ubica en un lugar determinante en el escenario de modernización del contenido de enseñanza, ya que señalan las diversas revoluciones pedagógicas entre nosotros, desde Lancaster hasta Decroly. Así lo confirman las siguientes expresiones:

Si las reformas a los sistemas de enseñanza en América empezaron con la introducción del modo mutuo o monitorial preconizado y propagado con el apoyo del Libertador en Venezuela, por Bell y Lancaster, más la introducción por medio de los alemanes en 1875 en la Normales del país de los métodos de Pestalozzi, basados en la intuición y en la contemplación directa de los objetos para oponerlos a los sistemas memoristas y verbalistas existentes, hecho este que constituyó la $2^{a}$ etapa de la revolución pedagógica entre nosotros; la tercera época dice el doctor Julio Cesar García, está marcada por la llegada del doctor Decroly a Colombia en 1925, quien visitó el país traído por el Gimnasio Moderno de Bogotá, Instituto este fundado por Dn. Agustín Nieto Caballero desde 1914 y donde ya se estaban practicando los principios fundamentales de la Escuela Activa, o lo que es lo mismo los fundamentos de la Escuela Nueva o funcional, tan nombrada hoy entre los educadores en Colombia. ${ }^{12}$

\section{Dos métodos de gran circulación: Decroly y Dewey}

\section{Los Centros de Interés. Decroly}

Durante la primera mitad del siglo XX, una de las rutas para modernizar las prácticas de enseñanza de los maestros colombianos fue mediante el programa del médico belga Ovidio Decroly, denominado "Centros de Interés". ${ }^{13}$ La apropiación de la Escuela Nueva en Colombia no se justifica porque los maestros habían leído todo lo relativo a la experiencia realizada por el Doctor Ovidio Decroly en Europa, sino además porque ellos hicieron ensayos parciales y generales para hacer uso del método de principios, más no de formulas, propuestos por Decroly, para la enseñanza de la lectura, la

\footnotetext{
12 José María. Rodriguez. “La Escuela Nueva de la Ladera. Anexa a la Normal de Institutores Medellín”, Tesis de grado para optar al título de maestro. Escuela Normal de Institutores de Antioquia, Medellín, 1938. 4.

13 Lorenzo Vanegas, "Alrededor de los Centros de Interés", Tesis de grado para optar al título de maestro. Escuela Normal de Institutores de Antioquia. Medellín, 1929. 1 También se puede consultar a Osiris Maldonado, "Los Centros de Interés. Medellín”, Tesis de grado para optar al título de maestro. Medellín, 1942..
} 
geometría, la aritmética, la matemática y las ciencias naturales. En 1938, el maestro Guillermo Luna redactó un ensayo sobre la enseñanza de la lectura usando los principios decrolyanos:

Tenemos, pues, que todos los procedimientos de la didáctica moderna tratan de establecer una cierta relación en la lectura inicial, relación que se efectúa entre los complejos de articulación y la imagen auditiva y la visual y con la imagen misma del ojo. Cuando se inicia la lectura, las imágenes del lenguaje hablado y los complejos motores de articulación, como también las imágenes de las cosas y hechos que el lenguaje simboliza tienen una representación la cual hay que aprender mediante los grafismos, para saber que el niño sí conoce las cosas. Como atrás se dice para representar las palabras hay de veinticuatro (24) a treinta y cinco (35) signos, más o menos, que corresponden a los sonidos en una misma cantidad, luego aprender a leer y a escribir es relacionar entre sí, ese número limitado de signos gráficos y articulados. La didáctica moderna afirma, además, que al dársele al niño la enseñanza no debe explicársele dicha relación, sino emplear otros medios adecuados para que la descubra él espontáneamente, utilizando como clave las cosas y los hechos que las palabras simbolizan. Mejor explicaremos con un ejemplo: Tomemos una naranja que se le presenta al niño y que él la reconoce como objeto representado, pues tiene la imagen de dicho fruta y pronuncia su nombre porque la conoce, porque posee la imagen auditiva y los complejos motores de articulación de la palabra. Si se presenta un dibujo con el objeto naranja, con un cartel donde aparezca dicho nombre y se le dice que la palabra [que] allí aparece es la misma del dibujo, el niño leerá inmediatamente la palabra naranja. Si se le dice que dibuje esa misma palabra dibujará los signos en un renglón seguido y reproducirá dichos signos escribiendo así la palabra naranja. Desde ese momento, haciendo una suficiente repetición interesante y adecuada, vienen a quedar unidos a las imágenes y complejos que poseía, (visual del objeto, auditiva de la palabra, complejo motor de articulación) la imagen visual gráfica y el complejo motor de la escritura; entonces viene a quedar cerrado el complejo sensorial y motor de la palabra naranja. ${ }^{14}$

Como se puede apreciar, para la enseñanza de la lectura se subrayaba la importancia del método ideovisual del Doctor Decroly que parte de las frases

14 Guillermo Luna, "La Lectura. Su importancia, valor educativo y social, lectura inicial etc., Tesis de grado para optar al título de maestro. Escuela Normal de Institutores de Antioquia. Medellín, 1938, 11. Arturo Tobón, "Método de Lectura Ideo Visual”, Tesis de grado para optar al título de maestro. Escuela Normal de Institutores de Antioquia. Medellín, 1938, 17. 
o ideas gráficas y va a las palabras. La novedad de este método radicaba en que la frase o imagen proporcionada al niño debía contener un significado, estableciendo una ruptura con los métodos de la pedagogía clásica. Además, este método cumplía con la función de globalización; se parte del todo -la frase-, y el análisis va sacando a la luz los componentes de la globalidad.

Por su parte, la enseñanza de la aritmética y la geometría entra también de lleno en la llamada enseñanza global o de las Ideas Asociadas propuesta por Decroly. Así lo demuestra el trabajo de grado del maestro Isaac Millán, en referencia a la enseñanza de la aritmética:

La aritmética es la ciencia que más se presta a subordinarse a los centros de interés por cuanto no hay motivo alguno en la vida que no podamos relacionar con problemas y cantidades. Los trabajos manuales se pueden enlazar intimamente con la enseñanza de las matemáticas. Una ciudad de cartón por ejemplo tendrá sus calles numeradas; una caja tendrá sus medidas, su volumen, su peso, etc. Si fabricamos una jaula, tendrá esta su valor de donde se podrá ver a base de cálculos, las posibilidades de una industria, teniendo en cuenta el precio de la materia prima y su valor en el comercio [...] El material que utilicemos para la enseñanza de los números, ya que debe empezarse y hasta donde sea posible continuarse a base de cosas concretas debe ser el que el mismo nos indique; sobre lo que para él sea interesante le daremos los conocimientos; si por ejemplo le gustó una colección de piedrecitas en el río, perfectamente la puede guardar y con ella el maestro podrá hacer ejercicios aritméticos (contar, sumar, restar, etc.). ${ }^{15}$

Para la enseñanza de las ciencias naturales los maestros seguían dos métodos, el inductivo y el experimental. El primero era utilizado con el argumento de que las ciencias naturales son materias constitutivas de la observación, primera etapa de los Centros de Interés del Doctor Decroly. El segundo, el experimental, consiste en la repetición del fenómeno en condiciones favorables para determinar cuáles son sus elementos esenciales y cuáles accesorios, estudiando con cuidado los primeros y prescindiendo de los últimos:

Con las Ciencias Naturales se responde mejor a las necesidades e intereses intelectuales de los niños, ya que según Spencer, la mayor parte de

15 Isaac Millán Bayona, "Metodología de la aritmética elemental”, Tesis de grado para optar al título de maestro. Escuela Normal de Institutores de Antioquia. Medellín, 1939, 11. Reinaldo Reales, "La enseñanza de la Geometría en la escuela primaria”, Tesis de grado para optar al título de maestro. Escuela Normal de Institutores de Antioquia. Medellín, 1933, 27. 
los niños son por instinto naturalistas. Por otra parte el estudio natural y experimental da a los niños hábitos de trabajo, agudiza el alcance de sus sentidos, despierta en ellos el sentido crítico, les da poder de auto-control, y les desarrolla el espiritu de investigación científica. Por el estudio de las Ciencias Naturales se pone al niño en contacto directo con la realidad, con la naturaleza y con la vida. Sus necesidades de obrar, crear, construir, encuentran en estos estudios el campo a propósito para desenvolverse. ${ }^{16}$

En suma, los maestros colombianos desarrollaron diversas prácticas para construir conocimiento didáctico a partir del método Ideo-Visual y los Centros de Interés, no solo para la enseñanza de la geometría, las ciencias naturales y la aritmética, sino también en Geografía y en muchos aspectos que afectaban la vida económica de nuestro país: la red de ferrovías, el carbón, y las vías fluviales de comunicación en Colombia como el río Atrato y el Amazonas, para conocer la riqueza natural de Colombia en la fauna y en la flora, además de sus límites con otros países.

¿Por qué Decroly, en el contexto general de la Escuela Nueva, fue particularmente apropiado en Colombia? Aunque para algunos historiadores de la educación en Colombia fue un acontecimiento anecdótico, para los maestros de la primera mitad del siglo XX fue un acontecimiento decisivo para la transformación de la escuela primaria en Colombia. Así lo prueban las siguientes expresiones:

Hacia el año de 1925 y traído por Agustín Nieto Caballero, visitó nuestras principales ciudades y centros de estudio el doctor Ovidio Decroly. Encierra de por sí esta visita, una época de transición cultural en el país, una era de progreso en nuestra educación, ya que el sabio pedagogo belga (nacido en Renaix en 1871) dejó con su palabra, con sus fervientes frases de hombre apóstol, científico y amante apasionado del alma infantil, honda huella en todos los espiritus cuyo amor al progreso nacional calcaron sus sistemas y empezaron a trabajar por la transformación de nuestra escuela. [...] Fue la visita del Doctor Decroly a Colombia de tanta influencia en los destinos de nuestra educación, que podemos decir, sin riesgo a equivocarnos, que fue entonces cuando nació verdaderamente la "Escuela Activa Colombiana [...]. ${ }^{17}$

16 Rodríguez, "La Escuela Nueva de la Ladera..." . 112. Gabriel Hoyos, "Las ciencias naturales en la escuela", Tesis de grado para optar al título de maestro. Escuela Normal de Institutores de Antioquia.Medellín, 1941, 27.

17 Emilio Bernal, "La escuela primaria en Colombia. Su evolución", Tesis de grado para optar al título de maestro. Escuela Normal de Institutores de Antioquia.Medellín, 1939, 28-29. 


\section{El método de proyectos: Dewey}

En el saber pedagógico colombiano John Dewey significó una discontinuidad con las concepciones sociales de corte evolucionista y determinista, las cuales sustentaron las reformas educativas de las primeras décadas del siglo XX en Colombia. Para Dewey era preciso en la educación rehacer la experiencia del niño pero no solo para adaptarlo a un ambiente determinado, por ejemplo al físico y social, o al espiritual de la raza, o al intelectual, sentimental y volitivo. Si para el belga Decroly el fin de la educación es la adaptación a las condiciones del medio natural y social, para Dewey la adaptación del niño no era un fin sino un medio. Es decir, el fin inmediato de la educación es el desarrollo de la capacidad para transformar el medio, superando las miradas que concebían el ambiente como algo fijo que proporcionaría la norma de los cambios en el organismo. ${ }^{18}$

Su concepción de la sociedad basada en un relativismo cultural, su preocupación por analizar los intereses sociales de la infancia y los problemas del medio social y cultural en el que se desenvuelve, su idea de conocimiento como la capacidad de adaptar el ambiente a nuestras necesidades, fines y deseos a la situación en que vivimos, así como también su criterio democrático para formar en el individuo hábitos como la libertad, la autonomía, la equidad, la participación y la tolerancia, fueron razones para que, en el saber pedagógico colombiano, la institución escolar, encontrara nuevos sentidos y lugares más allá del párroco, el alcalde y el maestro. A partir de las concepciones de Dewey sobre la democracia y la pedagogía social la escuela pasó a ser entendida por los maestros de las escuelas normales como una pequeña sociedad. De esta manera, la escuela desde su entorno social debía transformarse en una comunidad de vida embrionaria:

El viejo maestro no ha caído en la cuenta de que la Escuela es un organismo social en pequeño y que por lo tanto ha de relacionarse intimamente con el organismo social en grande. [...] ¿Qué son las instituciones escolares? Son todas aquellas actividades de la escuela que tienen un profundo significado en la acción social de la educación ya que son un medio propicio para aplicar el gran principio de Dewey: "el mundo

\footnotetext{
18 Para examinar con mayor detalle las diferencias entre las concepciones de Decroly y Dewey en Colombia, véase "Una pedagogía de lo social: de Decroly a Dewey", en Mirar la infancia: pedagogía, moral y modernidad en Colombia, 1903-1946, Volumen II, realizado por Javier Sáenz Obregón, Oscar Saldarriaga y Armando Ospina,, (Bogotá, Medellín: Colciencias- Ediciones Foro Nacional por Colombia- Ediciones Uniandes- Editorial Universidad de Antioquia, 1997), 320-394.
} 
pedagógico debe realizar la vida", es decir, que las actividades de la escuela deben estar guiadas u orientadas de un modo tal que se semejen a las de la comunidad social general. ${ }^{19}$

De ahí la importancia de las cooperativas escolares, las granjas escolares, colonias de vacaciones, como método de vida para fomentar "el espíritu de solidaridad en los niños, haciendo del trabajo de la escuela un medio y un resultado de la colaboración de todos los escolares". ${ }^{20} \mathrm{~A}$ lo que se agrega:

Fuera de las relaciones cordiales que produce la cooperativa entre los niños, estas se completarán con las establecidas entre ellos y el maestro, y entonces la escuela alcanzará esa clase de disciplina que Dewey defendia, "que nace de la escuela misma, de situaciones que exigen esfuerzo y que los niños consideran dignas de entrar en ellas". ${ }^{21}$

Por su parte, y como estrategia para erradicar los sistemas de enseñanza pasivos y tradicionales en la Escuela Normal Superior en Bogotá, se ensayó con alumnos del primer año de grado el método de proyectos del norteamericano John Dewey, tal y como nos lo confirma el Informe de labores de Francisco Socarrás en 1944:22

A raíz de mi ingreso al establecimiento, y por deseo expreso del Ministerio, se ensayó con alumnos de primer año el 'método de proyectos' de John Dewey. Los resultados fueron pocos satisfactorios, debido a la falta de preparación pedagógica de los profesores y, en especial, a la carencia de recursos materiales. ${ }^{23}$

No obstante, agrega Socarrás, que la experiencia les sirvió para reformar las prácticas de enseñanza tradicional que hacían aprender las lecciones de memoria. De esta manera, las enseñanzas de la casi totalidad de las asignaturas de la Normal se hicieron por medio de seminarios, investigaciones indivi-

19 Joaquín García, "La escuela y la sociedad", Tesis de grado para optar al título de maestro. Escuela Normal de Institutores de Antioquia Medellín, 1940, 5-6. También véase: Guillermo Arango, "La cooperación en la escuela", Tesis de grado para optar al título de maestro. Escuela Normal de Institutores de Antioquia , Bogotá, 1936. Bernardo Correa, "La función social de la escuela”, Tesis de grado para optar al título de maestro. Escuela Normal de Institutores de Antioquia. Medellín, 1944.

20 García, “La escuela y la sociedad”., 15-16.

21 Ibid., 20.

22 Vale la pena precisar que el método de proyectos no es de John Dewey, tal y como lo presenta Francisco Socarrás, sino fue creado por W.H. Kilpatrick en 1918. Lo fundó en el análisis del pensamiento hecho por John Dewey, y su cometido fue el ensayo de una forma más efectiva de enseñar. De los mismos principios que dieron origen al método de proyectos, surgió el método de problemas, por obra del propio Dewey.

23 José Socarrás, Facultades de Educación y Escuela Normal Superior, (Tunja: La Rana y el Águila, 1987), 39. 
duales, lecturas colaterales, fichas bibliográficas, resúmenes orales y escritos de libros y lecciones, prácticas de laboratorio, estudios de campo.

En suma, este grupo de perspectivas metodológicas, desarrolladas por los maestros en formación de las escuelas normales, permite hacer visible dos cuestiones, primero, los inicios de lo que sería un campo de la enseñanza de las ciencias y los saberes en Colombia. Campo de conocimiento acerca de la enseñanza que funciona sobre un asunto similar que constituye un tema central en la estructuración del pensamiento didáctico, a saber: el orden de la enseñanza. Bien sabemos que el problema del orden está planteado desde Comenio en el siglo XVI. Su obra marca los inicios sobre la organización de los contenidos en la enseñanza: cómo ordenar el decir, la exposición en la enseñanza, por dónde empezar, cuáles contenidos se preceden a otros, cómo conoce el hombre y cómo ha de enseñarse un saber. Segundo, que este proceso de apropiación de la Escuela Nueva en Colombia abre nuevos horizontes y elementos al problema del orden de la enseñanza. Más allá, del programa que fija el contenido de la enseñanza, se enfatiza en nuevos elementos como ordenadores de la enseñanza: necesidades, tendencias, instintos, que en una sola expresión se denominan los intereses del niño.

\section{CONCLUSIÓN}

De acuerdo con lo anterior, se puede afirmar que en Colombia la Escuela Nueva, con las propuestas de belga Decroly y el norteamericano Dewey, sí tuvo unos efectos transformadores en las concepciones sobre la escuela y la infancia y sobre las prácticas de enseñanza en las instituciones formadoras de maestros y la escuela primaria. Pero también, nos encontramos con diversas resistencias y limitaciones de naturaleza política, cultural y metodológica, para que estas nuevas concepciones se consolidaran por todo el territorio nacional. En primer lugar, la actitud nacionalista del gobierno nacional frente a las nuevas concepciones de origen europeo:

[...] que resultan inadecuados a las peculiaridades de nuestro pueblo e invariablemente propensos a desnacionalizarlo, a fomentar más aún esa tendencia colonial y mestiza que mueve a la casi totalidad de América a vivir fuera de sí misma y como anhelosa de borrarse sabe Dios qué culpas, con un baño lustral de europeísmo [...]. Si desea el gobierno poner un obstáculo arancelario a esa pedagogía de laboratorio que pe- 
riódicamente exportan Suiza, Bélgica o Alemania para delectación de los aficionados y confusión de la infancia y procura crear una escuela y una universidad colombianas para el servicio del hombre y la vida colombianos. $^{24}$

En segundo lugar, los padres de familia, en especial de las zonas rurales de Colombia, no confiaban en la nueva metodología de formación e instrucción que traían los maestros a sus escuelas. Al respecto Julio Cesar Feuillet, afirmaba:

La incomprensión de padres de familia, aferrados a las prácticas de la escuela tradicional en que se formaron [...] Así, por ejemplo, la enseñanza al aire libre la detestan, creyendo ellos que solo en el salón de clase, aprovechan los niños el tiempo! [...] Los trabajos de granja, las excursiones, los trabajos manuales, no son bien vistos hasta por las mismas autoridades escolares, convencidos que solamente en los textos es donde el niño puede aprender! ${ }^{25}$ [sic.]

Un análisis de la apropiación de la Escuela Nueva como ha tratado de mostrar este escrito no busca identificar las coherencias entre lo que los maestros leían y lo que efectivamente hacían. Es lo que se pone de presente, por ejemplo, en un Informe de Educación del Secretario de Educación Pública del Departamento de Antioquia, al manifestar preocupación por la escisión que observaba en los maestros entre un decir y un hacer que no correspondían:

No se puede negar que el magisterio antioqueño ha leído bastante literatura pedagógica producida en Bélgica, Francia o España. Casi no hay maestra rural que no nos hable de los "Centros de Interés", del "Método ideovisual de lectura", de la "Pedagogía de Decroly" o del "Método de Proyectos". No faltan en su conversación los métodos técnicos de psicología pedagógica y con frecuencia oímos que se habla de test, de baremos, ojivas y cosas más por el estilo. Cualquiera podría pensar que nos encontramos en el laboratorio de una escuela experimental. Pero qué desengaño se lleva el observador al darse cuenta de que en aquella escuela en donde se habla de "centros de interés" lo que más falta, precisamente, es el interés. [...] encontramos que solo una que otra unidad entiende bien aquello y que lo aplica convenientemente [...]

24 Darío Echandía, Memoria del Ministerio de Educación Nacional, (Bogotá: Imprenta Nacional, 193611.

25 Julio Cesar Feuillet, "La Escuela Activa", Tesis de grado para optar al título de maestro. Escuela Normal de Institutores de Antioquia Medellín, 1937. 
Vale decir que se posee mucha teoría, pero que es casi nada lo que se practica bien [...] Bien podría decirse que no hay maestro en Antioquia que desconozca el principio pedagógico de que "al niño hay que conocerlo". Pero son muy pocos los que entienden el verdadero sentido pedagógico de conocer. ${ }^{26}$

Aquí se pone de presente un modo para juzgar al maestro como un experimentador desprovisto de las herramientas necesarias para aplicar coherentemente las modernas teorías pedagógicas. Sin embargo, bien sabemos por otras fuentes documentales (memorias e informes de los ministros de Educación, y diversas formas que ensayaron los maestros en esta ruta) que los maestros de escuela fueron eje fundamental de este dispositivo de reforma y modernización del contenido de enseñanza, aunque esta parte de la historia de la enseñanza en Colombia es mal conocida, o desconocida del todo. Ya lo había dicho Durkheim en sus clases de pedagogía en la Universidad de la Sorbonne en París: “la historia de la enseñanza, cuando menos de la enseñanza nacional, es la primera de las propedéuticas para una cultura pedagógica". ${ }^{27}$

Finalmente, con esta investigación se pretende contribuir al conocimiento de los procesos de la modernización educativa en Colombia, por medio de la formación de los maestros como profesionales para la enseñanza de las disciplinas y de los saberes. En otros términos, abrir una brecha de reflexiones sobre la historia de los saberes y las disciplinas escolares por medio de las instituciones formadoras de los maestros. Esto nos obliga a problematizar la noción de disciplina escolar "que lejos de ser lo que se enseña y punto, una entidad sinónima de contenidos o de materias", ${ }^{28}$ representa una compleja relación entre pedagogía y ciencia. De esta manera, las tesis (o monografías) de grado de los maestros formados en las escuelas normales, entre otros registros documentales, representan un valioso registro para hacer visible una historia de la enseñanza de las disciplinas y los saberes en la escuela. Las tesis de los maestros constituyen un archivo pedagógico invaluable. Se puede decir que sin su examen no es posible comprender la complejidad de las prácticas de apropiación de los discursos y prescripciones peda-

26 Fidel Rodríguez, "Informe de la Secretaría de Educación Pública al Gobernador de Antioquia”, (Medellín: Archivo Histórico de Antioquia (AHA), 1937), f. 47.

27 Émile Durkheim, "Naturaleza y método de la pedagogía", en Educación y Sociología, editada por David A. Rincón Pérez (México: Ediciones Coyoacán, 2006) 85.

28 Aida Terrón Bañuelos y Pedro Alonso Velásquez, "La historia de las disciplinas escolares, una contribución esencial al conocimiento de la escuela. El caso de la Aritmética., Revista Complutense de Educación 10, 1 (1999): 306. 
gógicas. Como ningún otro tipo de documento, permite acercarse a la posición de sujeto del maestro dentro del campo de fuerzas que buscaban configurarlo, y las fintas, fugas y usos que hizo para lograr cierta autonomía, esto es, para crear.

Poco se ha avanzado en el análisis histórico sobre el maestro como sujeto de saber pedagógico en Colombia, cuando el problema se ha planteado por fuera de toda o con escasas referencias empíricas. En suma, se privilegiaron las tesis de grado de la Escuela Normal de Varones de Antioquia por haber sido la institución formadora de maestros, durante la primera mitad del siglo $\mathrm{XX}$, que más se destacó junto con otras dos escuelas normales por concentrar casi la mitad del alumnado normalista del país. Bien sabemos por el trabajo de Sáenz, Saldarriaga y Ospina que entre 1903 y 1946, las reformas de la educación pública se centraron en las escuelas de enseñanza primaria y en las instituciones formadoras de maestros. Los proyectos de reforma y la legislación educativa que los sustentaba buscaron redefinir la formación del maestro. En esa ruta, el maestro debía ser un especialista en los nuevos métodos de enseñanza.

\section{FUENTES}

Anzola Gómez, Gabriel. Metodología especial de enseñanza primaria. Bogotá: Librería Colombiana Camacho Roldán, 1939.

Anzola Gómez, Gabriel. Aspectos de la educación contemporánea. Bogotá: Librería Nueva, 1930

Archivo Pedagógico Escuela Normal Superior de Medellín (APENSM), Medellín - Colombia. Tesis. Esta noes una tesis de grado, es la ubicación física del archivo donde actualmente se encuentran las tesis de grado anteriormente citadas

Ministerio de Educación Nacional. Dirección de Educación Primaria. Programas de estudio de la naturaleza para la escuela primaria y observaciones metodológicas sobre la materia. Bogotá: Imprenta Nacional, 1940.

Rodríguez, Fidel. Informe de la Secretaría de Educación Pública al Gobernador de Antioquia. Medellín: Archivo Histórico de Antioquia (AHA), 1937.

\section{REFERENCIAS}

Dewey, John. Democracia y Educación. Buenos Aires: Editorial Losada, 1953.

Dewey, John. Los fines, las materias y los métodos de la educación (La educación y la democracia II). Madrid: Ediciones la Lectura, 1927. 
Dewey, John. Reconstrucción de la filosofía. Madrid: La Lectura, 1930.

Durkheim, Émile. “Naturaleza y método de la pedagogía”. En Educación y Sociología, editado por David A. Rincón Pérez México: Ediciones Coyoacán, 2006.

Foucault, Michel. Arqueología del saber. México: Siglo XXI, 1996.

Foucault, Michel. El gobierno de sí y de los otros. Curso en el Collège de France: 1982-1983. Buenos Aires: Fondo de

Cultura Económica, 2009.

Foucault, Michel. Nietzsche, La Genealogía, La Historia. Valencia, España: Pre-textos, 2000.

Herrera Cortés, Martha Cecilia. Modernización y Escuela Nueva en Colombia: 1914-1951. Bogotá: Plaza \& Janes Editores, 1999.

Quiceno, Humberto, Javier Sáenz Obregón y Luis Arturo Vahos. “La instrucción y la educación pública en Colombia: 1903-1997". En Modernización de los sistemas educativos Iberoamericanos siglo XX, Tomo II, editado por Olga Lucia Zuluaga Garcés y Gabriela Ossenbach Sauter. Bogotá: Editorial Magisterio, Colección Pedagogía e Historia, 2004.

Sáenz Obregón, Javier, Oscar Saldarriaga y Armando Ospina. Mirar la infancia: pedagogía, moral y modernidad en Colombia, 1903-1946, Tomo II. Bogotá, Medellín: Colciencias, Ediciones Foro Nacional por Colombia, Ediciones Uniandes, Editorial Universidad de Antioquia, 1997.

Saldarriaga Vélez, Oscar. Del oficio de Maestro. Prácticas y teorías de la pedagogía moderna en Colombia. Bogotá: Editorial Magisterio, 2003.

Socarrás, José Francisco. Facultades de Educación y Escuela Normal Superior. Tunja: La Rana y el Águila, 1987.

Terrón Bañuelos, Aida y Pedro Alonso Velásquez. "La historia de las disciplinas escolares, una contribución esencial al conocimiento de la escuela. El caso de la Aritmética.". Revista Complutense de Educación 10, 1 (1999): 305-333.

Zuluaga Garcés, Olga Lucia. Pedagogía e Historia. La historicidad de la pedagogía. La enseñanza un objeto de saber. Bogotá: Editorial Universidad de Antioquia-Anthropos-Siglo del Hombre Editores, 1999.

Zuluaga Garcés, Olga Lucia. "Prólogo" En Mirar la infancia: pedagogía, moral y modernidad en Colombia, 1903-1946, Tomo II, realizado por Javier Sáenz Obregón, Oscar Saldarriaga y Armando Ospina, XI-XXV. Bogotá, Medellín: Colciencias, Ediciones Foro Nacional por Colombia, Ediciones Uniandes, Editorial Universidad de Antioquia, 1997.

Zuluaga Garcés, Olga Lucia. Saber pedagógico y campos conceptuales. Bogotá: Idep, Proyecto de Investigación, 2005.

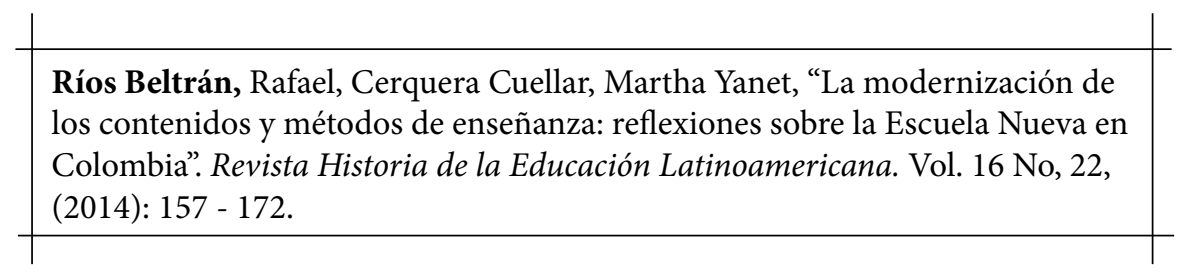

Rev. hist.educ.latinoam - Vol. 16 No. 22, enero - junio 2014 - ISSN: 0122-7238 - pp.157 - 172. 